\title{
Simulação realística como mediadora do processo ensino-aprendizagem na graduação em Farmácia: revisão sistemática
}

Realistic simulation as a mediator of the teaching-learning process in undergraduate Pharmacy: systematic review

Simulación realista como mediadora del proceso de enseñanza-aprendizaje en la carrera de Farmacia: revisión sistemática

\author{
Yolanda de Jesus Morais ${ }^{1}$ \\ ORCID: https://orcid.org/0000-0001-7105-8267 \\ Universidade Federal do Pará, Brasil \\ E-mail: yolandamorais123@gmail.com \\ Valéria Regina Cavalcante dos $\operatorname{Santos}^{1}$ \\ ORCID: https://orcid.org/0000-0002-1264-8125 \\ Universidade Federal do Pará, Brasil \\ E-mail: valregsantos@hotmail.com \\ Orenzio Soler ${ }^{1}$ \\ ORCID: http://orcid.org/0000-0003-2246-0019 \\ Universidade Federal do Pará, Brasil \\ E-mail: orenziosoler@gmail.com
}

\begin{abstract}
Resumo
Objetivo: sintetizar evidências sobre o uso da simulação realística como mediadora do processo ensino-aprendizagem na formação de farmacêuticos. Métodos: Revisão sistemática e síntese narrativa. Resultados: Os achados evidenciam que o uso da simulação realística como mediadora do processo ensino-aprendizagem no âmbito da educação de profissionais farmacêuticos proporciona benefícios ao desempenho acadêmico, aperfeiçoando o processo ensino-aprendizagem, tornando-o facilitado, contribuindo para o desenvolvimento de competências e habilidades na prática farmacêutica. Conclusão: Há evidências de que o uso da simulação realística proporciona a melhoria na satisfação, desempenho e segurança com o processo ensinoaprendizagem; assim como, em termos de competências e habilidades, a melhoria da gestão técnica da assistência farmacêutica, da gestão clínica do medicamento, da prática do cuidado farmacêutico, da resolução de problemas relacionados com medicamentos e da empatia pelos pacientes enquanto relações interpessoais.
\end{abstract}

Palavras-chave: Ensino. Educação farmacêutica; Plataformas de ensino; Simulação realística; Cuidado farmacêutico.

\begin{abstract}
Objective: to synthesize evidence on the use of realistic simulation as a mediator of the teaching-learning process in the training of pharmacists. Methods: Systematic review and narrative synthesis. Results: The findings show that the use of realistic simulation as a mediator of the teaching-learning process in the education of pharmaceutical professionals provides benefits to academic performance, improving the teaching-learning process, making it easier, contributing to the development of skills and skills in pharmaceutical practice. Conclusion: There is evidence that the use of realistic simulation provides an improvement in satisfaction, performance, and safety with the teaching-learning process; as well as, in terms of skills and abilities, the improvement of the technical management of pharmaceutical assistance, of the clinical management of the medicine, of the practice of pharmaceutical care, of the resolution of problems related to medicines and of empathy for patients as interpersonal relationships.
\end{abstract}

Keywords: Teaching; Pharmaceutical education; Teaching platforms; Realistic simulation; Pharmaceutical care.

\section{Resumen}

Objetivo: sintetizar evidencias sobre el uso de la simulación realista como mediador del proceso de enseñanza-aprendizaje en la formación de farmacéuticos. Métodos: Revisión sistemática y síntesis narrativa. Resultados: Los hallazgos muestran que el uso de la simulación realista como mediador del proceso de enseñanza-aprendizaje en la formación de los profesionales farmacéuticos aporta beneficios al desempeño académico, mejorando el proceso de enseñanza-aprendizaje, haciéndolo más fácil, contribuyendo al desarrollo de habilidades y habilidades en la práctica farmacéutica. Conclusión: Existe evidencia de que

\footnotetext{
${ }^{1}$ Programa de Pós-Graduação em Assistência Farmacêutica. Instituto de Ciências da Saúde. Universidade Federal do Pará.
} 
el uso de simulación realista proporciona una mejora en la satisfacción, desempeño y seguridad con el proceso de enseñanzaaprendizaje; así como, en cuanto a competencias y habilidades, la mejora de la gestión técnica de la atención farmacéutica, la gestión clínica de medicamentos, la práctica de la atención farmacéutica, la resolución de problemas relacionados con las drogas y la empatía por los pacientes como relaciones interpersonales.

Palabras clave: Enseñanza; Educación farmacéutica; Plataformas didácticas; Simulación realista; Cuidado farmacéutico.

\section{Introdução}

Nestes tempos de pós-modernidade, também identificado como da era digital ou da informação e interação social, fazse necessário repensar as metodologias de ensino utilizadas no cotidiano. O uso de novas tecnologias de aprendizagem e/ou técnicas de ensino possibilitam agregar maior conhecimento e tornar o dia a dia da sala de aula mais dinâmico, proporcionando a quebra do paradigma professor-aluno, uma vez que a velocidade da construção do conhecimento favorece a complementação destes personagens, que, cada vez mais, aprendem juntos.

\section{Teoria de aprendizagem experiencial}

Reconhece-se pedagogia como qualquer atividade consciente de uma pessoa projetada para aprimorar o aprendizado em outra. Refere-se tanto à teoria compreendida quanto à aplicação dessa teoria à prática de ensino que abrange todas as disciplinas, formas de compreensão e atividades de aprendizagem (Thompson, 2018).

Educação refere-se à perspectiva de um educador de que uma atividade específica que se destina a produzir uma mudança de conhecimento, habilidades ou atitudes pode realmente produzir uma mudança. Aprender é da perspectiva do aluno e implica um processo pelo qual o conhecimento é criado ou uma mudança nas habilidades ou atitudes são adquiridas (Thompson, 2018).

Existem várias teorias da aprendizagem estabelecidas na literatura que devem ser consideradas no desenvolvimento de novas ferramentas de aprendizagem. "Teorias comportamentalistas" se relacionam ao aprendizado por meio de recompensa ou punição e ter um processo mais passivo de aprendizado. "Teorias cognitivistas" estão relacionadas à aquisição, armazenamento e organização do conhecimento e a capacidade de um indivíduo aplicar o mesmo conhecimento para diferentes situações, incluindo processos de aprendizado passivos e ativos. "Teorias construtivistas" se referem a uma abordagem de aprendizagem mais centrada no aluno, na qual novas aprendizagens baseiam-se no conhecimento existente (Thompson, 2018).

Embora haja pouca evidência para mostrar que a aprendizagem em crianças e adultos difere uma da outra, o desenvolvimento de teorias de aprendizagem tem expressaram uma disparidade entre os dois. Um das mais utilizadas teorias construtivistas da aprendizagem é a Teoria de Aprendizagem Experiencial (Experiential Learning Theory - ELT) de Kolb (Kolb; Kolb, 2013). ELT é um modelo cíclico de aprendizado para adultos, composto por quatro estágios, que obedecem à teoria de que a aprendizagem é um processo pelo qual o conhecimento é criado através da transformação da experiência.

O ELT é uma perspectiva integrativa holística da aprendizagem que combina experiência, percepção, cognição e comportamento. A aprendizagem pode ser vista como um processo contínuo, fundamentado em experiência, na qual o conhecimento aprendido anterior pode e deve mudar com base em novas experiências (Kolb; Kolb, 2013).

No modelo de Kolb, o indivíduo é obrigado a progredir nas quatro etapas do ciclo para declarar que a aprendizagem efetiva ocorreu; experimentando, refletindo, pensando e agindo. Os indivíduos podem começar o ciclo em qualquer estágio, mas deve concluir a sequência para obter um ciclo completo de aprendizado. Idealmente, o ciclo de aprendizado experiencial deve começar com uma experiência concreta, de modo que um indivíduo na verdade, tenha uma experiência para aprender com ela, para incentivar o envolvimento ativo no processo de aprendizagem (Kolb; Kolb, 2013).

Essas experiências são a base de observações e reflexões que levam à conceitualização, interpretação e compreensão de relacionamentos. O estágio final é quando o aluno considera como eles vão aplicar seu aprendizado e criar experiências. 
Uma visão experiencial da aprendizagem onde os alunos são auto direcionados e capazes de assumir a responsabilidade por seus próprios processos de ensino-aprendizagem; capaz de refletir sobre sua própria prática de aprendizagem ao longo da vida (Kolb; Kolb, 2013).

\section{Aprendizado aprimorado pela tecnologia (e-Learning)}

A evolução tecnológica de computadores e disponibilidade para o uso na vida cotidiana vem direcionando o seu uso para apoiar a educação. O aprendizado aprimorado pela tecnologia (e-Learning) é descrito como um sistema baseado na Web que disponibiliza informações ou conhecimentos para usuários ou alunos e desconsidera restrições de tempo ou proximidade geográfica. Importante destacar o novo método de aprendizado chamado de m-Learning, que descreve a aprendizagem móvel e está se tornando cada vez mais popular na educação médica devido à portabilidade de dispositivos móveis. O e-Learning abrange diferentes ferramentas, incluindo Ambientes Virtuais de Aprendizagem (AVAs), Sistemas de Gerenciamento de Aprendizagem (LMS), Sistemas de Gerenciamento de Curso (CMS), ePortfolios, eAssessments e síncrona ou assíncrona ferramentas de comunicação (Thompson, 2018).

O aluno não deve mais ser visto como um ser passivo, mas estimulado a construir seu conhecimento por meio da avaliação da informação disponível, sendo o professor o responsável pela orientação adequada, pelo acompanhamento e pelo estímulo constante pelo aprendizado de qualidade. A utilização de recursos de aprendizagem em sala de aula deve contemplar o universo de ferramentas disponibilizadas pela Internet e softwares de computador, considerando que o aluno de hoje possui familiaridade com estes recursos, uma vez que grande parte os utiliza em suas atividades diárias de estudo, e, sobretudo, de entretenimento (Souza; Carvalho, 2010; Limberger, 2013; Brasil, 2014; 2017Lloyd et al., Brasil, 2017; 2018; Oliveira, 2019).

\section{Simulação realística}

Entre as variadas metodologias pedagógicas aplicadas na transmissão do conhecimento, a simulação realística ou de atendimento pode ser aplicada como parte de uma estratégia na aprendizagem da área da saúde, que promove um primeiro contato de alguns, com situações reais, mas realizada de forma assistida e hipotética, em um cenário montado, proporcionando a reprodução de eventos reais de maneira a dinamizar com o grupo presente, e efetivando a aprendizagem dos acadêmicos (Kolb; Kolb, 2013).

Em saúde, a simulação realística foi definida como uma técnica, não uma tecnologia, para substituir ou amplificar experiências reais com experiências, geralmente de natureza imersiva, que evocam ou replicam aspectos substanciais do mundo real de uma maneira totalmente interativa. As definições das diferentes ferramentas de simulação variam, mas as mais comumente implementadas na educação e no treinamento em saúde podem ser classificados como: pacientes padronizados ou simulados, simuladores de pacientes humanos, instruções assistidas por computador e pacientes virtuais (Thompson, 2018).

O termo "simulação" abrange uma variedade de ferramentas que podem ser classificadas de várias maneiras; a o mais comum é baseado na fidelidade das ferramentas ${ }^{(4)}$. Fidelidade refere-se ao grau de exatidão com que algo é copiado ou reproduzido e, portanto, refere-se ao retrato preciso e real da simulação. A simulação pode ser dividida em três categorias no base de fidelidade: 1) Baixa fidelidade - treinadores de tarefas parciais, simulação não computadorizada; 2) Fidelidade média pacientes padronizados, programas de computador, videogames, humano-paciente simulação; 3) Alta fidelidade - realidade virtual, simulação computadorizada de paciente humano (Thompson, 2018).

$\mathrm{O}$ argumento principal para a implantação de simulações no ensino superior na área da saúde, seria, à prática das habilidades clínicas sem danos ou riscos a pacientes. Na teoria, essas práticas de simulações, podem reduzir os erros cometidos na área profissional (Limberger, 2013; Thompson, 2018). 
Uma das vantagens da simulação é o ambiente ser controlado, com isso todos acadêmicos podem ser expostos e avaliados com as mesmas atividades e docentes, ou níveis de atividades semelhantes. $\mathrm{O}$ uso da simulação realística permite que todos os acadêmicos possam ter um feedback quase que instantâneo, podendo contar ainda com um ambiente monitorado para que eles possam rever suas atuações e se fazer uma autocrítica, podendo refletir e assim buscar uma melhora em seu desempenho (Limberger, 2013; Lloyd et al., 2018; Thompson, 2018).

\section{Formação farmacêutica}

O farmacêutico era um profissionalmente qualificado para desenvolver e realizar a logística do medicamento. Nos últimos anos, o papel do farmacêutico progrediu e vem integrando uma abordagem mais clínica e centrada no paciente; ou seja, trabalhando o cuidado farmacêutico. Hoje os farmacêuticos trabalham principalmente em farmácias comunitárias e/ou hospitalares, tendo novas atribuições na equipe de saúde. A integração na equipe de multidisciplinar é cada vez mais enfatizada na profissão farmacêutica, em especial na Atenção Primária à Saúde; uma mudança apoiada por iniciativas governamentais; ou seja, sobre o Exercício das Atividades Farmacêuticas nas Farmácias Comunitárias (Brasil, 2014) e das Diretrizes Curriculares Nacionais do Curso de Graduação em Farmácia (Brasil, 2017; Ferreira \& Soler, 2020).

Neste contexto, os cursos de Graduação em Farmácia vêm passando por distintas modificações na sua estruturação e organização, tendo como propósito formar profissionais farmacêuticos, que atuem nas mais diversificadas áreas da saúde; em especial na prestação de serviços técnicos-gerenciais e/ou técnico-assistenciais, integrados às equipes multiprofissionais, mantendo contato direto com a comunidade; em especial com o utente (Limberger, 2013; Thompson, 2018).

As simulações realísticas veem sendo utilizadas como estratégia no processo ensino-aprendizagem, treinamentos e ponderação de técnica de aptidões; uma ferramenta nova e sofisticada, empregando simuladores pacientes virtuais, intérpretes profissionais e demais personagens para a reprodução de ocasiões cotidianas e reais em uma área física adequada, contextualizada na atividade peculiar sugerida, onde os partícipes podem interatuar em certo período, além de motivar a reflexão em grupos a respeito dos desfechos evidenciados (Lloyd et al., 2018; Oliveira, 2019).

Trata-se de avanços bem-vindos para a profissão farmacêutica, onde o amplo conhecimento da eficácia dos fármacos, efetividade dos medicamentos e eficiência dos tratamentos são essenciais para garantir a segurança e a melhoria da qualidade de vida do paciente. A educação e a formação de farmacêuticos de graduação e pós-graduação precisam estar alinhadas com as mudanças do perfil profissional para garantir que os alunos estejam qualificados com habilidades e competências parar a práxis farmacêutica.

Neste contexto, o objetivo deste trabalho foi sintetizar evidências sobre o uso da simulação realística como mediadora do processo ensino-aprendizagem na formação de farmacêuticos.

\section{Método}

Trata-se de uma Revisão Sistemática (Brasil, 2012; Ercole; Melo; Alcoforado, 2014; Silva; Azevedo, 2019), tendo como questão norteadora a existência de evidências sobre o uso da simulação realística como mediadora do processo ensinoaprendizagem na formação acadêmica de farmacêuticos, tendo como desfechos a melhoria do processo ensino-aprendizagem, do desempenho acadêmico e do desenvolvimento de competências e habilidades. O recorte temporal foi de 2015 a 2019. Buscou-se estudos publicados nas bases Cochrane Library, Epistemonikos, Health Evidence, Health Systems Evidence, Biblioteca Virtual de Saúde e Google Scholar. Para a estratégia de busca, utilizou-se os descritores "Pharmacy education" e "Realistic simulation", adaptados ao Medical Subject Headings (MeSH) e Descritores em Ciências da Saúde (DeCS), associados com operadores boleanos "AND" e "OR". Restringiu-se a pesquisa as línguas portuguesa, espanhola e inglesa. 
Utilizou-se como critérios de inclusão: População: Alunos de graduação em Farmácia; Intervenção: Uso de simulação realística; Controle: Não utilização da simulação realística ou qualquer outra metodologia ativa; Desfecho: melhoria do processo ensino-aprendizagem, do desempenho acadêmico e de competências e habilidades desenvolvidas. Tipo de estudos: Estudo transversal, Relato de experiência, Revisão Integrativa, Revisão Sistemática. Não foram avaliados a qualidade dos artigos selecionados.

Foram recuperados 187 registros, sendo 2 da BVS, 1 da Cochrane, 8 do Epistemonikos, 3 do Health Evidence, 2 do Health Systems Evidence, 7 do NCBI, 164 do Google Scholar e 4 registros identificados por outras fontes. Foram removidas 104 duplicatas, sendo ao final selecionados 16 artigos (Figura 1). Os resultados estão apresentados por meio de síntese narrativa.

Figura 2 - Fluxograma da seleção dos artigos incluídos

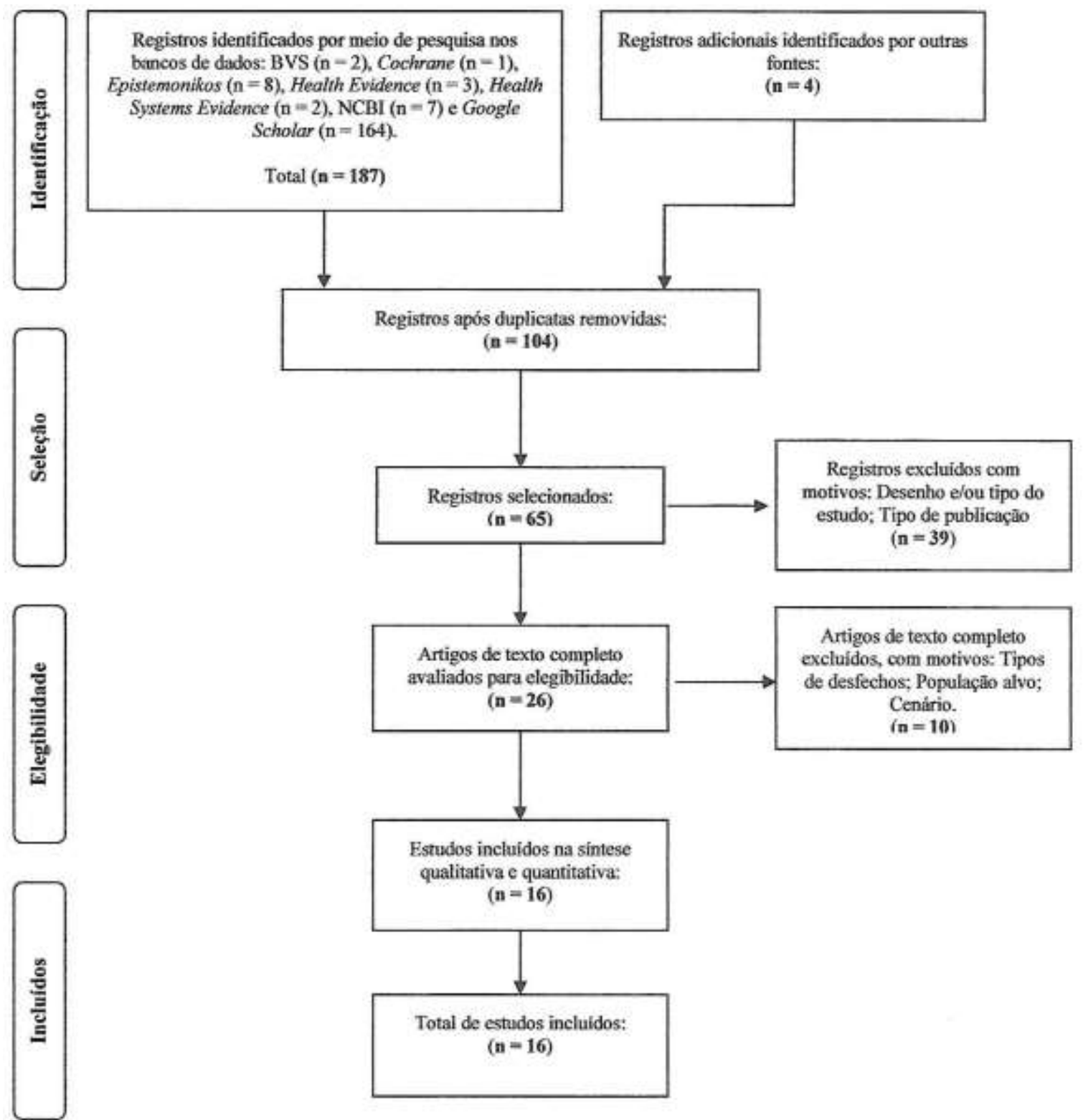

Fonte: Adaptado de Moher et al. (2009). 


\section{Resultados e discussão}

16 artigos foram selecionados, sendo 1 de revisão sistemática e 15 estudos transversais. O perfil e características dos estudos estão apresentados na Tabela 1.

Os achados evidenciam que o uso da simulação realística como mediadora do processo ensino-aprendizagem no âmbito da educação de profissionais farmacêuticos proporciona benefícios ao desempenho acadêmico, aperfeiçoando o processo ensino-aprendizagem, tornando-o facilitado, contribuindo para o desenvolvimento de competências e habilidades na prática farmacêutica (Tabela 2).

Katoue et al., (2019), Parker et al., (2019), Richardson et al., (2019), Ambrozia et al., (2018), Bernaitis et al., (2018), Lynch et al., (2018), Ong et al., (2018), Bastin et al., (2017), Ferrone et al., (2017), Taglieri et al., (2017), Barnett et al., (2016) e Curley et al., (2016) apresentaram evidências quanto ao processo ensino-aprendizagem e desempenho acadêmico relacionadas a melhoria no envolvimento com o processo ensino-aprendizagem, da satisfação e desempenho com o processo ensino-aprendizagem e da segurança no processo ensino-aprendizagem.

Richardson et al., (2019), Ambrozia et al., (2018), Bastin et al., (2017) e Daupin et al., (2016) apresentaram evidências quanto a competências e habilidades relacionadas a melhoria da gestão técnica da assistência farmacêutica.

Parker et al., (2019), Richardson et al., (2019), Bernaitis et al., (2018), Lynch et al., (2018), Bastin et al., (2017), Taglieri et al., (2017), Barnett et al., (2016), Daupin et al., (2016), Thompson (2016) e Kerr et al., (2015) apresentaram evidências quanto a competências e habilidades relacionadas a melhoria da gestão clínica do medicamento.

Katoue et al., (2019), Parker et al., (2019), Richardson et al., (2019), Taglieri et al., (2017), Barnett et al., (2016), Curley et al., (2016), Daupin et al., (2016), Fejzic et al., (2016), Thompson (2016) e Kerr et al., (2015) apresentaram evidências quanto a competências e habilidades relacionadas a melhoria da prática do cuidado farmacêutico.

Katoue et al., (2019), Parker et al., (2019), Richardson et al., (2019), Ambrozia et al., (2018), Taglieri et al., (2017), Barnett et al., (2016), Curley et al., (2016), Daupin et al., (2016), Thompson (2016) e Kerr et al., (2015) apresentaram evidências quanto a competências e habilidades relacionadas a melhoria da resolução de problemas relacionados com medicamentos.

Parker et al., (2019), Richardson et al., (2019), Curley et al., (2016), Thompson (2016) e Kerr et al., (2015) apresentaram evidências quanto a competências e habilidades relacionadas a melhoria da empatia pelos pacientes.

Tabela 1 - Perfil e características dos artigos selecionados.

\begin{tabular}{|c|c|c|c|}
\hline ARTIGO & OBJETIVO & $\begin{array}{l}\text { DESENHO DO } \\
\text { ESTUDO } \\
\text { TÉCNICA DE } \\
\text { SIMULAÇÃO }\end{array}$ & DESFECHO \\
\hline Katoue et al., (2019). & $\begin{array}{l}\text { Explorar as percepções dos } \\
\text { farmacêuticos sobre o uso de } \\
\text { simulação na educação profissional } \\
\text { continuada. }\end{array}$ & $\begin{array}{l}\text { Estudo } \\
\text { Transversal. } \\
\text { Pacientes Virtuais } \\
\text { (PVs). }\end{array}$ & $\begin{array}{l}\text { Um seminário de educação continuada baseado em simulação } \\
\text { foi bem recebido pelos farmacêuticos e aprimorou o } \\
\text { conhecimento, nível de satisfação e o conhecimento para a } \\
\text { reconciliação de medicamentos. São necessários esforços para } \\
\text { expandir o uso da simulação realística no desenvolvimento, } \\
\text { aprimoramento e manutenção das habilidades clínicas dos } \\
\text { farmacêuticos durante toda sua vida acadêmica. }\end{array}$ \\
\hline Parker et al., (2019). & $\begin{array}{l}\text { Avaliar o impacto de uma atividade } \\
\text { de simulação na empatia dos alunos } \\
\text { do curso de farmácia em relação aos } \\
\text { pacientes com diabetes. }\end{array}$ & $\begin{array}{l}\text { Estudo } \\
\text { Transversal. } \\
\text { Pacientes Virtuais } \\
\text { (PVs). }\end{array}$ & $\begin{array}{l}\text { Os resultados da pesquisa pré e pós-atividade (Diabetes } \\
\text { Simulation Survey Questions Posed) indicaram mudanças } \\
\text { estatisticamente significativas nas respostas relacionadas à } \\
\text { empatia. Essa simulação realística para o manejo do diabetes } \\
\text { aumentou a empatia e a confiança dos alunos no } \\
\text { gerenciamento de aspectos selecionados á farmacoterapia. }\end{array}$ \\
\hline
\end{tabular}




\begin{tabular}{|c|c|c|c|}
\hline $\begin{array}{l}\text { Richardson et al., } \\
\text { (2019). }\end{array}$ & $\begin{array}{l}\text { Realizar uma sistemática revisão } \\
\text { narrativa para estabelecer e avaliar } \\
\text { o uso de Pacientes Virtuais (PVs) } \\
\text { em farmácia. Isso incluiu PVs que } \\
\text { foram usados para desenvolver ou } \\
\text { contribuir para habilidades de } \\
\text { comunicação ou aconselhamento } \\
\text { graduandos de farmácia, } \\
\text { farmacêuticos de pré-registro } \\
\text { farmacêuticos qualificados. }\end{array}$ & $\begin{array}{l}\text { Revisão } \\
\text { sistemática } \\
\text { Pacientes Virtuais } \\
\text { (PVs). }\end{array}$ & $\begin{array}{l}\text { Os Pacientes Virtuais são um recurso adicional valioso para } \\
\text { desenvolver habilidades de comunicação e aconselhamento } \\
\text { para estudantes de farmácia. Estudos complementares são } \\
\text { requeridos para diferentes tecnologias e projetos de ensino, } \\
\text { assim como padrões de qualidade podem ajudar a contribuir } \\
\text { para o desenvolvimento harmonizado para ser aplicado em } \\
\text { distintas profissões. }\end{array}$ \\
\hline $\begin{array}{l}\text { Ambrozia et al., } \\
(2018) \text {. }\end{array}$ & $\begin{array}{l}\text { Verificar se a simulação virtual é } \\
\text { usada em um ambiente realista e } \\
\text { seguro para os estudantes } \\
\text { farmacêuticos aprenderem e } \\
\text { praticarem uma variedade de } \\
\text { habilidades nos contextos didático e } \\
\text { experimental. }\end{array}$ & $\begin{array}{l}\text { Estudo } \\
\text { Transversal. } \\
\text { Pacientes Virtuais } \\
\text { (PVs). }\end{array}$ & $\begin{array}{l}\text { O Programa de Simulação Virtual My Dispense permitiu que } \\
\text { os alunos identificassem a quantidade de prática que julgavam } \\
\text { necessária para obter habilidades específicas relacionadas à } \\
\text { gestão de medicamentos. }\end{array}$ \\
\hline $\begin{array}{l}\text { Bernaitis et al., } \\
(2018) \text {. }\end{array}$ & $\begin{array}{l}\text { Determinar os benefícios de casos } \\
\text { baseados em computador para } \\
\text { terapêutica oncológica em termos } \\
\text { de satisfação e desempenho do } \\
\text { aluno. }\end{array}$ & $\begin{array}{l}\text { Estudo } \\
\text { Transversal. } \\
\text { Pacientes Virtuais } \\
\text { (PVs). }\end{array}$ & $\begin{array}{l}\text { Uma simulação em computador para farmacoterapêutica } \\
\text { oncológica pode envolver os alunos e desenvolver habilidades } \\
\text { de tomada de decisão. O DecisionSim }{ }^{\mathrm{TM}} \text { aumentou a } \\
\text { satisfação e o desempenho do aluno no gerenciamento de } \\
\text { casos clínicos oncológicos, sendo uma ferramenta educacional } \\
\text { benéfica para ensinar tópicos terapêticos complexos a } \\
\text { estudantes de farmácia. }\end{array}$ \\
\hline Lynch et al., (2018). & 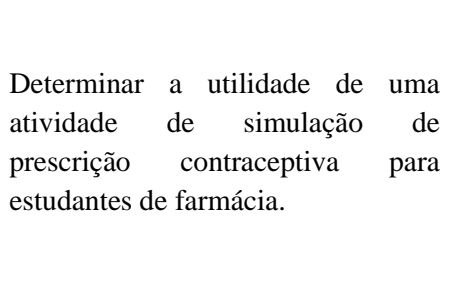 & $\begin{array}{l}\text { Estudo } \\
\text { Transversal. } \\
\text { Pacientes Virtuais } \\
\text { (PVs). }\end{array}$ & $\begin{array}{l}\text { A atividade de simulação apresentou aos alunos do curso de } \\
\text { farmácia, o processo de prescrição usando formas e cenários } \\
\text { realistas. Introduziu os alunos ao ambiente em constante } \\
\text { mudança da prática de farmácia, permitindo que os estudantes } \\
\text { aplicassem seus conhecimentos sobre contraceptivos e a saúde } \\
\text { da mulher. Os alunos tiveram um bom desempenho nas } \\
\text { atividades e relataram altos níveis de satisfação. }\end{array}$ \\
\hline Ong et al., (2018). & $\begin{array}{l}\text { Avaliar a aceitação de alunos do } \\
\text { curso de farmácia sobre o uso de } \\
\text { simulação de pacientes humanos de } \\
\text { alta fidelidade em comparação com } \\
\text { a aprendizagem on-line assíncrona. }\end{array}$ & $\begin{array}{l}\text { Estudo } \\
\text { Transversal. } \\
\text { Pacientes Virtuais } \\
\text { (PVs). }\end{array}$ & $\begin{array}{l}\text { A maioria dos participantes sentiram que a atividade de } \\
\text { simulação melhorou suas habilidades críticas e de tomada de } \\
\text { decisão, registrando que a atividade de simulação melhorou a } \\
\text { confiança. A atividade de simulação pode ser usada para obter } \\
\text { melhores habilidades críticas e de tomada de decisão dos } \\
\text { alunos. O aprendizado usando simulação de pacientes } \\
\text { humanos de alta fidelidade foi bem recebido pelos } \\
\text { participantes e pode ser implementado na educação } \\
\text { profissional continuada. }\end{array}$ \\
\hline Bastin et al., (2017). & $\begin{array}{l}\text { É descrito o uso do treinamento de } \\
\text { simulação de alta fidelidade para } \\
\text { preparar os residentes de farmácias } \\
\text { para várias emergências médicas de } \\
\text { alto estresse e alto impacto e o } \\
\text { impacto desse treinamento na } \\
\text { percepção de preparação dos } \\
\text { residentes de farmácias. }\end{array}$ & $\begin{array}{l}\text { Estudo } \\
\text { Transversal. } \\
\text { Pacientes Virtuais } \\
\text { (PVs). }\end{array}$ & $\begin{array}{l}\text { O treinamento em Programa de Simulação de Pacientes } \\
\text { Humanos de Alta Fidelidade (HPS) aumentou as competências } \\
\text { e habilidades de residentes de Farmácias para cenários clínicos } \\
\text { de alto estresse, alto impacto e emergências médicas. }\end{array}$ \\
\hline $\begin{array}{l}\text { Ferrone } \\
(2017) .\end{array}$ & $\begin{array}{l}\text { O objetivo deste estudo foi adaptar } \\
\text { colaborativamente um programa de } \\
\text { simulação internacional existente } \\
\text { para utilidade nos EUA e medir a } \\
\text { percepção dos alunos de um } \\
\text { programa de simulação de farmácia } \\
\text { comunitária baseado na Web em } \\
\text { três escolas de farmácia dos EUA. }\end{array}$ & $\begin{array}{l}\text { Estudo } \\
\text { Transversal. } \\
\text { Pacientes Virtuais } \\
\text { (PVs). }\end{array}$ & $\begin{array}{l}\text { A maioria dos estudantes concordou fortemente que o My } \\
\text { Dispense era fácil de aprender, mais realista quando } \\
\text { comparados a casos semelhantes em papel, oferecendo } \\
\text { oportunidades de aprendizado e aumento da segurança. O My } \\
\text { Dispense permitiu aos alunos praticar como coletar } \\
\text { informações do paciente e fazer perguntas apropriadas, } \\
\text { aconselhar pacientes e praticar o processo de cuidado } \\
\text { farmacêutico. O uso da simulação realística é positiva e } \\
\text { oferece uma opção viável para a introdução e o reforço de } \\
\text { habilidades de prática de farmácia comunitária para os alunos }\end{array}$ \\
\hline
\end{tabular}




\begin{tabular}{|c|c|c|c|}
\hline & & & durante a educação em farmácia. \\
\hline $\begin{array}{l}\text { Taglieri et al., } \\
(2017) \text {. }\end{array}$ & $\begin{array}{l}\text { Avaliar o efeito da incorporação de } \\
\text { atividades virtuais do paciente em } \\
\text { um laboratório de habilidades } \\
\text { farmacêuticas na competência e } \\
\text { confiança do aluno ao realizar } \\
\text { visitas clínicas abrangentes em } \\
\text { tempo real com pacientes } \\
\text { simulados. }\end{array}$ & $\begin{array}{l}\text { Estudo } \\
\text { Transversal. } \\
\text { Pacientes Virtuais } \\
\text { (PVs). }\end{array}$ & $\begin{array}{l}\text { O Shadow Health and Advanced Health Assessment (Digital } \\
\text { Clinical Experience) proporcionou a melhoria do desempenho } \\
\text { dos alunos durante visitas simuladas à clínica. Os alunos } \\
\text { sentiram que o paciente virtual simulava realisticamente um } \\
\text { paciente real. Pacientes virtuais podem oferecer oportunidades } \\
\text { adicionais de aprendizado para os alunos. }\end{array}$ \\
\hline $\begin{array}{l}\text { Barnett et al., } \\
(2016) \text {. }\end{array}$ & $\begin{array}{l}\text { Avaliar a simulação de caso on-line } \\
\text { versus um caso de papel sobre a } \\
\text { confiança e o envolvimento dos } \\
\text { alunos. }\end{array}$ & $\begin{array}{l}\text { Estudo } \\
\text { Transversal. } \\
\text { Pacientes Virtuais } \\
\text { (PVs). }\end{array}$ & $\begin{array}{l}\text { As simulações de casos virtuais resultam em maior } \\
\text { envolvimento dos alunos, podendo levar a um melhor } \\
\text { desempenho. Os casos de pacientes virtuais podem oferecer } \\
\text { um benefício limitado sobre os casos em papel, melhorando a } \\
\text { autoconfiança geral dos alunos para fornecer gerenciamento } \\
\text { de medicamentos. }\end{array}$ \\
\hline Curley et al., (2016). & $\begin{array}{l}\text { Explorar as perspectivas dos } \\
\text { estudantes de farmácia em relação à } \\
\text { integração de uma comunidade } \\
\text { virtual em um curso de graduação } \\
\text { em farmácia no terceiro ano. }\end{array}$ & $\begin{array}{l}\text { Estudo } \\
\text { Transversal. } \\
\text { Pacientes Virtuais } \\
\text { (PVs). }\end{array}$ & $\begin{array}{l}\text { A Plataforma Virtual de Ensino denominada NZ Pharmville } \\
\text { para o curso de Farmácia foi avaliada como positiva. As } \\
\text { respostas indicaram que muitos dos alunos consideraram a } \\
\text { integração da comunidade virtual uma preparação útil para as } \\
\text { sessões práticas, sendo que a maioria dos estudantes achou } \\
\text { que as vinhetas tornavam mais fácil o desenvolvimento da } \\
\text { empatia pelos pacientes em vez de ler sobre eles. }\end{array}$ \\
\hline $\begin{array}{l}\text { Daupin et al., } \\
(2016) \text {. }\end{array}$ & $\begin{array}{l}\text { Avaliar a capacidade da equipe } \\
\text { médica, de enfermagem e de } \\
\text { farmácia de identificar erros } \\
\text { relacionados ao sistema de uso de } \\
\text { medicamentos usando uma } \\
\text { simulação e avaliar também o nível } \\
\text { de satisfação dos envolvidos. }\end{array}$ & $\begin{array}{l}\text { Estudo } \\
\text { Transversal. } \\
\text { Pacientes Virtuais } \\
\text { (PVs). }\end{array}$ & $\begin{array}{l}\text { A simulação realista de erros no sistema de infusão de } \\
\text { medicamentos em um hospital materno-infantil foi uma } \\
\text { ferramenta eficaz, relevante e inovadora para aumentar a } \\
\text { conscientização dos profissionais de saúde (incluindo os } \\
\text { farmacêuticos) sobre processos críticos de administração de } \\
\text { medicamentos. }\end{array}$ \\
\hline Fejzic et al., (2016). & $\begin{array}{l}\text { Examinar a eficácia dos módulos de } \\
\text { aprendizado simulado (SLMs) que } \\
\text { abrangem as competências } \\
\text { essenciais do EXcellence in } \\
\text { Learning e Liderança Experimental } \\
\text { Cultural (EXCELL) no } \\
\text { aprimoramento das habilidades de } \\
\text { comunicação profissional dos } \\
\text { estudantes de farmácia. }\end{array}$ & $\begin{array}{l}\text { Estudo } \\
\text { Transversal. } \\
\text { Pacientes Virtuais } \\
\text { (PVs). }\end{array}$ & $\begin{array}{l}\text { O feedback dos alunos foi positivo sobre os Módulos de } \\
\text { Aprendizado Simulado (SLM) como uma ferramenta de } \\
\text { aprendizado eficaz. A maioria apresentou novas áreas de } \\
\text { aprendizagens e constatou que o EXcellence in Cultural } \\
\text { Experiential Learning and Leadership (EXCELL) aprimorou } \\
\text { suas habilidades e confiança profissionais. }\end{array}$ \\
\hline Thompson (2016). & $\begin{array}{l}\text { Explorar as perspectivas dos } \\
\text { farmacêuticos de pré-registro sobre } \\
\text { a utilidade e a usabilidade dos } \\
\text { pacientes virtuais (PVs) como uma } \\
\text { ferramenta de treinamento. }\end{array}$ & $\begin{array}{l}\text { Estudo } \\
\text { Transversal. } \\
\text { Pacientes Virtuais } \\
\text { (PVs). }\end{array}$ & $\begin{array}{l}\text { Os acadêmicos de farmácia relataram que o Pacientes Virtuais } \\
\text { (PVs) contribuiu para desenvolver um amplo conjunto de } \\
\text { habilidades e base de conhecimento. }\end{array}$ \\
\hline Kerr et al., (2015). & $\begin{array}{l}\text { Avaliar o impacto de uma } \\
\text { simulação de interação de paciente / } \\
\text { provedor de } 6 \text { semanas nos níveis } \\
\text { de empatia e autoeficácia das } \\
\text { habilidades de gerenciamento de } \\
\text { diabetes em estudantes de farmácia } \\
\text { do terceiro ano. }\end{array}$ & $\begin{array}{l}\text { Estudo } \\
\text { Transversal. } \\
\text { Pacientes Virtuais } \\
\text { (PVs). }\end{array}$ & $\begin{array}{l}\text { A simulação realística de paciente/cuidador melhorou os } \\
\text { níveis de empatia e autoeficácia em estudantes do terceiro ano } \\
\text { de farmácia. }\end{array}$ \\
\hline
\end{tabular}

Fonte: Simulação realística como mediadora do processo ensino-aprendizagem na graduação em Farmácia (2020). 
Research, Society and Development, v. 10, n. 10, e241101018783, 2021

(CC BY 4.0) | ISSN 2525-3409 | DOI: http://dx.doi.org/10.33448/rsd-v10i10.18783

Tabela 2 - Desfechos de simulações realísticas realizadas no processo ensino-aprendizagem para farmácia.

\begin{tabular}{|c|c|c|c|c|c|c|c|c|}
\hline \multirow[b]{3}{*}{ ARTIGO } & \multicolumn{8}{|c|}{ DESFECHOS } \\
\hline & \multicolumn{3}{|c|}{ Processo ensino-aprendizagem e desempenho acadêmico } & \multicolumn{5}{|c|}{ Competências e habilidades } \\
\hline & $\begin{array}{l}\text { Melhoria no } \\
\text { envolvimento com } \\
\text { o processo ensino- } \\
\text { aprendizagem }\end{array}$ & $\begin{array}{c}\text { Melhoria da } \\
\text { satisfação e } \\
\text { desempenho com o } \\
\text { processo ensino- } \\
\text { aprendizagem }\end{array}$ & $\begin{array}{c}\text { Melhoria da } \\
\text { segurança no } \\
\text { processo ensino- } \\
\text { aprendizagem }\end{array}$ & $\begin{array}{l}\text { Melhoria da } \\
\text { gestão técnica } \\
\text { da assistência } \\
\text { farmacêutica }\end{array}$ & $\begin{array}{l}\text { Melhoria da } \\
\text { gestão clínica } \\
\text { do } \\
\text { medicamento }\end{array}$ & $\begin{array}{l}\text { Melhoria da } \\
\text { prática do } \\
\text { cuidado } \\
\text { farmacêutico }\end{array}$ & $\begin{array}{l}\text { Melhoria da } \\
\text { resolução de } \\
\text { problemas } \\
\text { relacionados } \\
\text { com } \\
\text { medicamentos }\end{array}$ & $\begin{array}{l}\text { Melhoria da } \\
\text { empatia } \\
\text { pelos } \\
\text { pacientes e } \\
\text { relações } \\
\text { interpessoais }\end{array}$ \\
\hline Katoue et al., (2019) & $(+)$ & $(+)$ & $(+)$ & & & $(+)$ & $(+)$ & \\
\hline Parker et al., (2019) & $(+)$ & $(+)$ & $(+)$ & & $(+)$ & $(+)$ & $(+)$ & $(+)$ \\
\hline Richardson et al., (2019) & $(+)$ & $(+)$ & $(+)$ & $(+)$ & $(+)$ & $(+)$ & $(+)$ & $(+)$ \\
\hline Ambrozia et al., (2018) & $(+)$ & $(+)$ & $(+)$ & $(+)$ & & & $(+)$ & \\
\hline Bernaitis et al., (2018) & $(+)$ & $(+)$ & $(+)$ & & $(+)$ & & & \\
\hline Lynch et al., (2018) & $(+)$ & $(+)$ & $(+)$ & & $(+)$ & & & \\
\hline Ong et al., (2018) & $(+)$ & $(+)$ & $(+)$ & & & & & \\
\hline Bastin et al., (2017) & $(+)$ & $(+)$ & $(+)$ & $(+)$ & $(+)$ & & & \\
\hline Ferrone et al., (2017) & $(+)$ & $(+)$ & $(+)$ & & & & & \\
\hline Taglieri et al., (2017) & $(+)$ & $(+)$ & $(+)$ & & $(+)$ & $(+)$ & $(+)$ & \\
\hline Barnett et al., (2016) & $(+)$ & $(+)$ & $(+)$ & & $(+)$ & $(+)$ & $(+)$ & \\
\hline Curley et al., (2016) & $(+)$ & $(+)$ & $(+)$ & & & $(+)$ & $(+)$ & $(+)$ \\
\hline
\end{tabular}


Research, Society and Development, v. 10, n. 10, e241101018783, 2021

(CC BY 4.0) | ISSN 2525-3409 | DOI: http://dx.doi.org/10.33448/rsd-v10i10.18783

\begin{tabular}{|c|c|c|c|c|c|c|c|c|}
\hline Daupin et al., (2016) & & & & $(+)$ & $(+)$ & $(+)$ & $(+)$ & \\
\hline Fejzic et al., (2016) & $(+)$ & $(+)$ & $(+)$ & & & $(+)$ & & \\
\hline Thompson (2016) & $(+)$ & $(+)$ & $(+)$ & & $(+)$ & $(+)$ & $(+)$ & $(+)$ \\
\hline Kerr et al., (2015) & $(+)$ & $(+)$ & $(+)$ & & $(+)$ & $(+)$ & $(+)$ & $(+)$ \\
\hline
\end{tabular}

Legenda: ( ) = Referências (artigos pesquisados) e suas evidências. (-) = Não foi estudado e/ou não há evidências.

Fonte: Simulação Realística como mediadora do processo ensino-aprendizagem na graduação em Farmácia, 2020. 
Katoue et al., (2019) informam que a simulação é uma valiosa técnica de ensino para a aquisição, aprimoramento e manutenção das habilidades clínicas dos farmacêuticos durante toda sua formação profissional; tanto na graduação quanto para a Educação Continuada em Farmácias (ECF). Realizaram um workshop de ECF baseado em simulação realística que resultou em alto nível de satisfação e melhorias de competências. O uso efetivo da simulação realística em ECF exige equipamentos adequados, educadores treinados e a institucionalização curricular. Integrar o uso da simulação realística em ECF parece ser desafiador. Entretanto, investimentos de recursos financeiros para o treinamento e aprimoramento do corpo docente e a aquisição de software, facilitam a incorporação na cultura e missão das instituições de ensino de saúde, contribuindo para superar esses desafios na implementação da simulação em processo de ECF.

Parker et al., (2019) registraram que a atividade de simulação realística impactou positivamente a empatia dos alunos com pacientes com diabetes. A confiança do aluno na gestão clínica do diabetes melhorou significativamente.

Richardson et al., (2019) informam que conhecimentos e habilidades, confiança, e satisfação com o aprendizado melhoraram. O uso de Pacientes Virtuais é vantajoso, mas problemas tecnológicos podem limitar seu uso. Os Pacientes Virtuais contribuem para a transição do conhecimento teórico e prático.

Ambrozia et al., (2018) registram que os alunos avaliaram que o uso da simulação realística é uma ferramenta eficaz para aprender habilidades quanto a gestão técnica da assistência farmacêutica e gestão clínica do medicamento. A maior barreira à implementação foi a o tempo necessário para estruturar e testar cada exercício.

Bernaitis et al., (2018) demonstraram os benefícios e a viabilidade do aprendizado resultantes da introdução do DecisionSim $^{\mathrm{TM}}$, um software para simulação realística para o estudo da farmacoterapia oncológica aplicado a estudantes de Farmácia. A estratégia de ensino foi bem recebida pelos estudantes, permitindo o aprimoramento de conhecimentos teóricos e o desenvolvimento de habilidades e competências quanto a tomada de decisão em terapêutica oncológica. Os resultados se apoiam na satisfação dos alunos.

Lynch et al., (2018) projetaram simulação realística para ensinar os alunos a fazer a gestão clínica de contraceptivos hormonais, uma área nova para a maioria dos farmacêuticos. A estratégia se mostrou eficaz. Ao final do treinamento, os alunos se mostraram confiantes e satisfeitos. Preconizam que a simulação realística possa ser incorporada nos currículos de Farmácia.

Ong et al., (2018) registram que a Aprendizagem Baseada em Simulação de Paciente Humano de Alta Fidelidade (HPS) foi bem recebida pelos alunos durante em evento de Educação Profissional Continuada (EPC). Relatam que a Aprendizagem On-Line Assíncrona (AOL); um aplicativo gratuito, pode ser um ambiente de aprendizado interessante para o HPS.

Bastin et al., (2017) demonstram que o treinamento em simulação realística é uma ferramenta eficaz para aumentar o conhecimento, a satisfação e a segurança de alunos de quanto ao trabalho em cenários clínicos de emergências médicas de alto estresse e alto impacto. $\mathrm{O}$ treinamento em simulação realística pode ser personalizado para atender às necessidades de categorias profissionais específicas.

Ferrone et al., (2017) apresentam o trabalho de adaptação e customização do software australiano MyDispense ${ }^{\mathrm{TM}}$, incorporada com sucesso nos currículos de três Faculdades de Farmácia dos Estados Unidos da América (EUA). O software tem capacidade de criar exercícios quase ilimitados usando distintos parâmetros e indicadores em diferentes cenários e combinações.

Taglieri et al., (2017) avaliaram um aplicativo de Pacientes Virtuais (PVs) como inovação curricular e a melhoria do desempenho dos alunos em gestão clínica de medicamentos. Os achados informam um desempenho melhor dos estudantes na gestão clínica de medicamentos para agravos cardiovascular e pulmonar. A simulação realística fornece oportunidades de aprendizado adicionais para os alunos e melhoram seu desempenho. 
Barnett et al., (2016) registram aumento significativo quanto a confiança dos alunos relacionada ao cuidado farmacêutico. A simulação realística aumentou a compreensão da relevância, interesse, prazer e realismo.

Curley et al., (2016) estudaram o aplicativo Pharmville NZ ${ }^{\mathrm{TM}}$, demonstrando ser promissor como plataforma para o aprimoramento do ensino-aprendizagem. O Pharmville NZ ${ }^{\mathrm{TM}}$ permite aos alunos acesso contínuo a videoclipes de pacientes que descrevem uma situação da vida real, permitindo que os alunos se envolvam com os personagens fictícios. Foi bem recebida pelos alunos, permitindo um aprendizado ativo centrado no paciente, reflexionando semanalmente sobre suas habilidades quanto ao cuidado farmacêutico.

Daupin et al., (2016) implementaram e testaram um aplicativo de simulação realista quanto a erros no uso de medicamentos em ambiente hospitalar materno-infantil. O feedback dos participantes foi muito positivo e a relevância da simulação foi amplamente elogiada. As simulações são uma forma eficaz e eficiente de implementar, ensinar ou atualizar as melhores práticas e procedimentos clínicos. Ensino inovador de conhecimento sobre sistemas complexos e processos críticos do manuseio de medicamentos são necessários para a conscientização dos profissionais de saúde.

Fejzic et al., (2016) estudaram o processo de inovação curricular quando da inclusão de Módulos de Aprendizado Simulado (SLMs) que incorporam princípios do EXcellence in Cultural Experiential Learning and Leadership (EXCELL) no curso de Farmácia. Houve um feedback positivo e encorajador de alunos e funcionários. Os SLMs que se adaptam às necessidades dos alunos em formação e/ou aperfeiçoamento de profissionais, são uma estratégia pedagógica eficaz. Sua força reside em permitir que os alunos adquiram habilidades que eles podem usar na sala de aula e práxis profissional. Os aumentaram sua confiança, competências e habilidades profissionais.

Thompson (2016) avaliou a eficácia da simulação realística utilizando aplicativos de Pacientes Virtuais (PVs) versus aplicativos de Aprendizado Não Interativo (ANI). Os estudos de caso utilizando Pacientes Virtuais (VPs) foram associados a maior satisfação e propiciou uma experiência de aprendizado mais realista, interativa e agradável. O uso do VPs favorece o desenvolvimento de habilidades e competências quanto aos complexos aspectos da vida real.

Kerr et al., (2015) demonstraram a autoeficácia dos estudantes para a gestão do medicamento, interação e a empatia com pacientes diabéticos usando a simulação realística utilizando Pacientes Virtuais (VPs). A confiança dos alunos na sua capacidade de fazer a gestão clínica de medicamentos em pacientes com diabetes aumentou em todos os aspectos; em especial nas entrevistas e no exame físico do paciente.

Apesar da distinção de aplicativos disponíveis, os alunos melhoram o conhecimento, a confiança, habilidades e competência. A simulação realística por meio de Pacientes Virtuais (PVs), permite oportunidades para a prática; uma vantagem bem discutida na literatura. Qualquer que seja o objetivo dos PVs, benefícios particulares são que eles podem fornecer aprendizado ricamente contextualizado e aplicados na prática, de tal modo que o aluno possa aprender com segurança. Isso está alinhado com o aprendizado experimental, onde o foco está em investigações conduzidas por alunos, geralmente em busca de uma tarefa real ou artificial (Kolb; Kolb, 2013; Thompson, 2018).

Ao usar os PVs no processo ensino-aprendizagem experimental, os alunos estão em um ambiente de aprendizado ativo onde eles são capazes de reflexionar sobre seu próprio aprendizado, através de investigação, resolução de problemas e novas descobertas. Isso foi explicitamente mencionado pelos autores, que registram a importância da aprendizagem experimental por meio de simulações realísticas; isto é, experiências práticas. Os PVs são capazes de fornecer experiências novas e seguras para os alunos, colocando-os a reflexionar sua práxis e a refinar os modelos teóricos. Os PVs também permitem que o aluno se autoavalie quanto a sua aprendizagem e foque na sua aprendizagem pessoal (Kolb; Kolb, 2013; Limberger, 2013; Thompson, 2018).

Para que novos PVs sejam aprimorados, é necessário um nível de conhecimento sobre a aprendizagem experiencial e designers. Ao usar aplicativos, há oportunidades de simular cenários clínicos onde o aluno pratica, crie confiança e aumente 
sua precisão quanto a tomada de decisões. Oportunidades práticas acessíveis, padronizadas, seguras e confiáveis são os benefícios do PVs relatado com consistência nos estudos apresentados (Limberger, 2013; Lloyd; Watmough; Bennett, 2018; Oliveira, 2019).

Reconhece-se, contudo, dificuldades que precisam ser consideradas no desenvolvimento e aprimoramento de futuros aplicativo de simulações realísticas. A tecnologia; ou seja, os aplicativos devem ser acessíveis, de fácil manejo e manutenção. O local da simulação realística dentro de um currículo também precisa ser cuidadosamente observado. É importante que os princípios pedagógicos e educacionais sejam garantidos quando da utilização do PVs (Kolb; Kolb, 2013; Limberger, 2013; Thompson, 2018).

Por fim, a simulação ideal tenta obter uma alta fidelidade, o suficiente para convencer os usuários de que eles estão usando algo que se assemelha ao que eles encontrariam na vida real. As simulações clínicas devem imitar o ambiente clínico e permitir que o aluno aplique habilidades cognitivas, afetivas e psicomotoras em um cenário realista, sem induzir emoções e efeitos negativos.

\section{Limitações e viés}

Utilizou-se rigorosa pesquisa para identificar e fornecer uma síntese da literatura atualizada sobre evidências do uso da simulação realística como mediadora do processo ensino-aprendizagem na formação de farmacêuticos. Entretanto, há possibilidade de se ter perdido artigos potencialmente elegíveis devido as distintas sinonímias de descritores chaves. Há estudos de pequena escala, com limitações e sem resultados robustos.

Os autores dos estudos selecionados alertam para se ter cautela com a interpretação dos resultados, especialmente em face da dificuldade de agregar dados e da heterogeneidade dos estudos quanto a variação, tipos, intensidade e multiplicidade de indicadores e uso de síntese narrativa, uma vez que não há metanálises.

\section{Conclusão}

Há evidências de que o uso da simulação realística proporciona a melhoria na satisfação, desempenho e segurança com o processo ensino-aprendizagem; assim como, em termos de competências e habilidades, a melhoria da gestão técnica da assistência farmacêutica, da gestão clínica do medicamento, da prática do cuidado farmacêutico, da resolução de problemas relacionados com medicamentos e da empatia pelos pacientes enquanto relações interpessoais.

\section{Implicações práticas}

Recomenda-se a necessidade de estudos complementares quanto a regulamentação de padrões mínimos para a aplicação prática de simulação realística no contexto do processo ensino-aprendizagem. Seria oportuno, também, estudos econômicos para aferir a relação custo-eficácia e custo-benefício dos aplicativos disponíveis no mercado.

\section{Contribuições dos autores}

YJM concebeu a estratégia de pesquisa em conjunto com OS e VRCS. YJM fez as buscas bibliográficas nas bases de dados, a seleção dos artigos e a extração de dados sob a supervisão de OS e VRCS. Os autores leram e aprovaram a versão final do manuscrito. O conteúdo da revisão é de exclusiva responsabilidade individuais dos autores.

\section{Declaração de conflito de interesses}

Os autores declaram que a pesquisa foi conduzida na ausência de quaisquer relações comerciais ou financeiras que possam ser interpretados como um potencial conflito de interesse. 


\section{Referências}

Ambroziak, K., Ibrahim, N., Marshall, V.D. \& Kelling, S. E. (2018). Virtual simulation to personalize student learning in a required pharmacy course. Currents in Pharmacy Teaching and Learning. 10(6):750-756. https://doi.org/10.1016/j.cptl.2018.03.017

Barnett, S. G., Gallimore, C. E., Pitterle, M. \& Morrill, J. (2016). Impact of a paper vs virtual simulated patient case on student-perceived confidence and engagement. American Journal of Pharmaceutical Education. (1):16 doi: DOI: 10.5688/ajpe80116

Bastin, M. L., Cook, T. A. M. \& Flannery, A. H. (2017). Use of simulation training to prepare pharmacy residents for medical emergencies. American Journal of Health-System Pharmacy. 74(6):424-429. DOI 10.2146/ajhp160129

Bernaitis, N., Baumann-Birkbeck, L., Alcorn, S., Powell, M., Arora, D. \& Anoopkumar-Dukie, S. (2018). Simulated patient cases using DecisionSim ${ }^{\mathrm{TM}}$ improves student performance and satisfaction in pharmacotherapeutics education. Currents in Pharmacy Teaching and Learning. 10(6):730-735. https://doi.org/10.1016/j.cptl.2018.03.020

Brasil. (2012). Ministério da Saúde. Secretaria de Ciência, Tecnologia e Insumos Estratégicos. Departamento de Ciência e Tecnologia. Diretrizes metodológicas: elaboração de revisão sistemática e metanálise de ensaios clínicos randomizados. Brasília: Editora do Ministério da Saúde, 2012. 92 p.: il. (Série A: Normas e Manuais Técnicos). ISBN 978-85-334-1951-3

Brasil. (2014). Presidência da República. Lei $\mathrm{n}^{\circ}$ 13.021, de 8 de agosto de 2014. Dispõe sobre o exercício das atividades farmacêuticas nas farmácias comunitárias. Brasília. Distrito Federal. Publicado no DOU em 11/8/2014.

Brasil. (2017). Ministério da Educação. Conselho Nacional de Educação, Câmara De Educação Superior. Resolução nº 6, de 19 de outubro de 2017. Institui as Diretrizes Curriculares Nacionais do Curso de Graduação em Farmácia. Brasília. Distrito Federal. Publicado no DOU em: 20/10/2017. Edição: 202. Seção: 1. Página: 30

Curley, L. E., Mc Donald, M. \& Aspden, T. (2016). Use of a fictitious community-based virtual teaching platform to aid in the teaching of pharmacy practice skills: Student perspectives after initial implementation. Curley et al. Journal of Pharmaceutical Policy and Practice. 9:24. DOI 10.1186/s40545-016-0077-3

Daupin, J., Atkinson, S., Bédard, P., Pelchat, V., Lebel, D. \& Bussières, J. F. (2016). Medication errors room: a simulation to assess the medical, nursing and pharmacy staffs' ability to identify errors related to the medication-use system. Journal of evaluation in clinical practice. 22(6):911-920. ISSN1365-2753

Fejzic, J., Barker, M., Hills, R. \& Priddle, A. (2016). Communication capacity building through pharmacy practice simulation. American journal of pharmaceutical education. 80(2):1-11. ISSN: 0002-9459 (print); 1553-6467 (web).

Ferreira, A. S. \& SOLER, Orenzio. Fortalecimento das estratégias de segurança de pacientes: uma revisão integrativa quantos aos processos de segurança de medicamentos. Research, Society and Development. 9(12): e129129564, 2020. (CC BY 4.0) | ISSN 2525-3409 | DOI: http://dx.doi.org/10.33448/rsdv9i12.9564

Ferrone, M., Kebodeaux, C., Fitzgerald, J. \& Holle, L. (2017). Implementation of a virtual dispensing simulator to support US pharmacy education. Currents in Pharmacy Teaching and Learning. 9(4):511-520. http://dx.doi.org/10.1016/j.cptl.2017.03.018

Ibrahim, M. I., Palaian, S., Al-Sulaiti, F. \& El-Shami, S. (2016). Evaluating community pharmacy practice in Qatar using simulated patient method: acute gastroenteritis management. Pharmacy Practice (Granada). 14(4):1-8. doi: 10.18549/PharmPract.2016.04.800

Katoue, M. G. \& Ker, J. (2019). Simulation for Continuing Pharmacy Education: Development and Implementation of a Simulation-Based Workshop on Medicines Reconciliation for Pharmacists. Journal of Continuing Education in the Health Professions. 39(3):185-193. DOI: 10.1097/CEH.0000000000000257

Kerr, J. L., Stahnke, A. M. \& Behnen, E. M. (2015). Assessing empathy and self-efficacy levels of pharmacy students in an elective diabetes management course. American Journal of Pharmaceutical Education. 79(3)Article 42. ISSN: 0002-9459 (print); $1553-6467$ (web).

Kolb, A. \& Kolb, D. (2013). The kolb learning style inventory - Version 4.0 A Comprehensive Guide to the Theory, Psychometrics, Research on Validity and Educational Applications. Experience Based Learning Systems, Inc. Experience Based Learning Systems. www.learningfromexperience.com

Limberger, J. B. (2013). Metodologias ativas de ensino-aprendizagem para educação farmacêutica: um relato de experiência. Interface (Botucatu) [Internet]. 17(47):969-975. http://dx.doi.org/10.1590/1807-57622013.3683.

Lloyd, M., WAtmough, S. \& Bennett, N. (2018). Simulation-based training: applications in clinical Pharmacy. Clinical Pharmacist. 10(9). DOI: 10.1211/CP.2018.20205302

Lynch, S. E., Griffin, B. L. \& Vest, K. M. (2018). Assessment of a simulated contraceptive prescribing activity for pharmacy students. Currents in Pharmacy Teaching and Learning. 10(2):178-184. http://dx.doi.org/10.1016/j.cptl.2017.10.013

Moher, D., Liberati, A., Tetzlaff, J. \& Altman, D.G. (2009). The PRISMA Group. Preferred Reporting Items for Systematic Reviews and Meta-Analyses: The PRISMA Statement. PLoS Med. 6(7):e1000097. DOI:10.1371/journal.pmed1000097

Oliveira, V. G. (2019). Proposta de sala para simulação realística de práticas e serviços farmacêuticos. Ariquemes. Trabalho de Conclusão de Curso (TCC). Curso de Graduação em Farmácia. Faculdade de Educação e Meio Ambiente. Rondônia.

Ong, C. L., Kane-Gill, S. L., Kobulinsky, L. R, Hon, J. S., Kong, M. C. \& Seybert, A.L. (2018). Evaluation of pharmacist satisfact

ion with simulation-based learning in Singapore. Currents in Pharmacy Teaching and Learning. 10(10):1414-1418. https://doi.org/10.1016/j.cptl.2018.07.004 Parker, D., Fontem, A., Ojong, E. \& Pope, J. (2019). Impact of diabetes simulation on empathy in pharmacy students. American Journal of Pharmaceutical Education. 83(1):69-72. Article 6432. ISSN: 0002-9459 (print); 1553-6467 (web). 
Research, Society and Development, v. 10, n. 10, e241101018783, 2021

(CC BY 4.0) | ISSN 2525-3409 | DOI: http://dx.doi.org/10.33448/rsd-v10i10.18783

Richardson, C. L., White, S. \& Chapman. S. (2019). Virtual patient technology to educate pharmacists and pharmacy students on patient communication: a systematic review. BMJ Stel. 0:1-7. doi:10.1136/bmjstel-2019-000514

Silva, A. R. \& Azevedo, M. S. (2019). Revisão sistemática: uma aplicação metodológica. REASU - Revista Eletrônica de Administração da Universidade Santa Úrsula. 3(2):1-15. http://revistas.icesp.br/index.php/REASU/article/view/435

Taglieri, C. A., Crosby, S. J., Zimmerman, K., Schneider, T. \& Patel, D. K. (2017). Evaluation of the use of a virtual patient on student competence and confidence in performing simulated clinic visits. American journal of pharmaceutical education. 81(5):1-9. Article 87. ISSN: 0002-9459 (print); 1553-6467 (web).

Thompson, J. (2018). Clinical simulations using virtual patient avatars for pre-registration pharmacist training: a mixed methods evaluation. Staffordshire. Thesis (Doctor of Philosophy). Keele University. United Kingdom.

Thompson, J. F. (2016). A qualitative study of the perspectives of pre-registration students on the use of virtual patients as a novel educational tool. The International Journal of Pharmacy Practice. 24(11):34-34. ISSN: 0961-7671; 2042-7174 